\title{
Infección por Mycobacterium scrofulaceum en un niño de las Islas Galápagos - Ecuador
}

\author{
Raif Nasre-Nasser y Greta Muñoz-López'
}

\section{Mycobacterium scrofulaceum infection in a pediatric patient from the Galapagos Islands - Ecuador}

Mycobacterium scrofulaceum is a slow-growing atypical mycobacteria with a reservoir in the environmen, and occasionally it causes disease in humans. The case of a 10 -year-old patient with fever of four months of evolution is presented. Symptoms include bilateral adenopathies of neck, thorax, abdomen and inguinal region, rash, abdominal pain and vomiting; in addition, a positive serology for EBV and a culture of the left inguinal ganglia with growth of $M$. scrofulaceum were evidenced. Thus, this is the first report of human infection caused by the aforementioned mycobacterium in Ecuador, which it was particularly manifested with an unusual pattern of resistance.

Keywords: Lymphadenopathy, Mycobacterium scrofulaceum, Epstein Barr virus, pediatrics, Ecuador.

Palabras claves: Linfadenopatías, Mycobacterium scrofulaceum, virus de Epstein Barr, pediatría, Ecuador.

\section{Introducción}

M ycobacterium scrofulaceum es un bacilo aerobio inmóvil catalogado como una micobacteria atípica escotocromógena de crecimiento lento (clasificación de Runyon II) ${ }^{1,2}$. El principal reservorio de M. scrofulaceum es el ambiente (aguas superficiales templadas, aguas marinas, suelo y polvo), aunque ha sido aislado de ganglios linfáticos y heces de animales domésticos y salvajes ${ }^{3}$. Se asocia ocasionalmente con enfermedades en los seres humanos, siendo las adenopatías cervicales en niños y la enfermedad pulmonar en adultos, la presentación clínica más frecuente. No obstante, existen reportes de lesiones cutáneas y enfermedad diseminada, generalmente asociadas a estados de inmunodepresión ${ }^{2}$.

Se presenta el caso de un niño con linfadenopatías generalizadas en que se demostró una infección por $M$. scrofulaceum y Epstein Barr.

\section{Caso clínico}

Varón de 10 años de edad, procedente de las Islas Galápagos, sin antecedentes personales ni familiares de importancia y con esquema de inmunizaciones completo para su edad. Inició cuadro de fiebre hasta $40^{\circ} \mathrm{C}$, edema y dolor en la rodilla izquierda, dos días después de haber sufrido un traumatismo local, con erosiones que entraron en contacto con el agua de mar. Por persistencia de la fiebre a pesar de la regresión de los signos inflamatorios, se diagnosticó una probable infección urinaria, recibiendo amoxicilina y gentamicina durante 5 días. El paciente evolucionó con fiebre, compromiso del estado general, adenomegalias y mialgias generalizadas y artralgias de manos y rodillas.

Se realizaron pruebas de antígenos febriles (Salmonella enterica Typhi y Paratyphi, Proteus mirabilis, Brucella abortus) y serologías (IgM, IgG) para toxoplasmosis, paludismo, leptospirosis, virus de hepatitis A y B, virus del dengue, virus chikungunya, citomegalovirus y VIH, que resultaron negativas. La IgM anti-cápside de VEB (por técnica de ELISA) fue positiva, con IgG negativa. Los títulos de antiestreptolisina O (ASO) fueron bajos (100 U/ml), PPD fue $0 \mathrm{~mm}$ y el ensayo de liberación de interferón resultó negativo. Los hemocultivos y cultivo de LCR también fueron negativos.

Persistió con fiebre intermitente por cuatro meses, agregándose dolor abdominal, hiporexia y vómitos ocasionales. Además presentó un exantema pruriginoso intermitente inicialmente en la región axilar y crural y luego en la región posterior del cuello.

Desde el inicio del cuadro clínico el paciente presentó leucocitosis entre 18.000 y 30.000 cél $/ \mathrm{mm}^{3}$, con desviación a la izquierda y presencia de células inmaduras. Los resultados de la biopsia de médula ósea fueron compatibles con cambios secundarios a un proceso inflamatorio y/o infeccioso. El ecocardiograma fue normal. En la ecografía de la región inguinal se visualizaron múltiples adenopatías izquierdas de aspecto inflamatorio. En una TC se observaron adenopatías cervicales (submandibulares y supraclaviculares bilaterales), axilares bilaterales e intraabdominales (mesentéricas y retroperitoneales). Se realizó una tomografía por emisión de positrones (PET-CT, por sus siglas en inglés) que mostró ganglios
'Hospital Pediátrico Baca Ortiz, Quito, Ecuador.

Los autores declaran no tener conflictos de interés.

Fuente de financiamiento: ninguna.

Recibido: 14 de marzo de 2018 Aceptado: 23 de octubre de 2018

Correspondencia a: Greta Muñoz-López gretaml@yahoo.es 


\section{Caso Clínico}

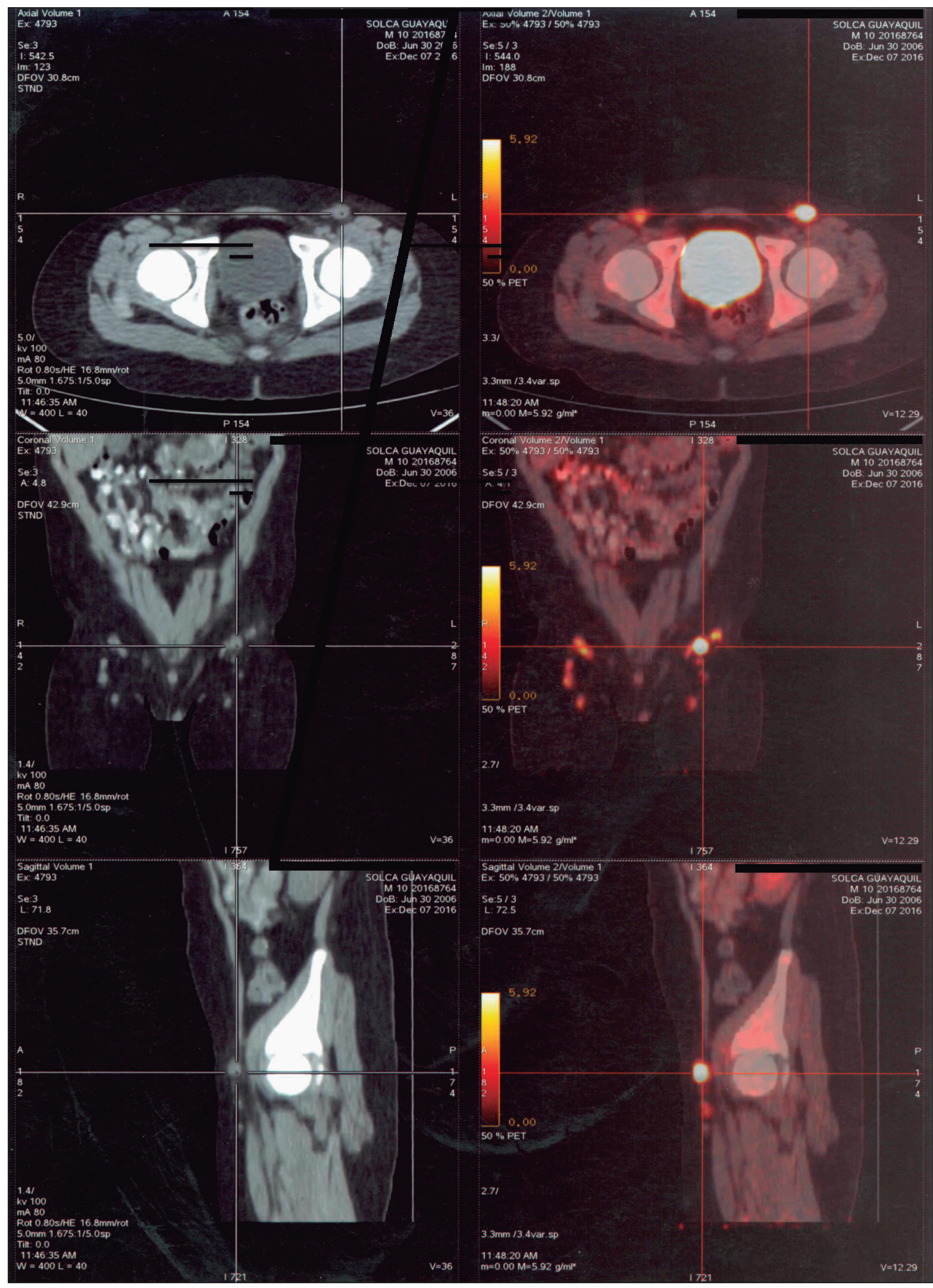

Figura 1. Tomografía por emisión de positrones segmento lumbosacro. Se observan ganglios hipermetabólicos a nivel inguinofemoral superficiales y profundos de hasta $12 \mathrm{~mm}$ y en región ilíaca externa bilateral menores a $10 \mathrm{~mm}$. Fuente: Servicio de imagenología del hospital de SOLCA Guayaquil. 
bilaterales hipermetabólicos en región cervical, axilar, retropectoral, abdominal, región ilíaca externa e inguinofemoral superficiales y profundos, entre 6 a $12 \mathrm{~mm}$ y un valor de captación estándar (SUV, por sus siglas en inglés) entre 2,8 y 17,7; con el mayor valor de captación en los ganglios inguinales (Figura 1). En el cintigrama óseo no se evidenciaron tumores o lesiones óseas metastásicas.

Debido a la alta actividad metabólica evidenciada en el PET y por la facilidad para el abordaje quirúrgico, se realizó exéresis de los ganglios inguinales izquierdos para análisis histopatológico y para investigación de tuberculosis (en el laboratorio de referencia nacional para el diagnóstico de micobacterias del Instituto Nacional de Investigación en Salud, Quito, Ecuador).

Con el diagnóstico de síndrome febril prolongado y linfoadenopatías hipermetabólicas generalizadas ingresó a nuestro hospital para continuar con el estudio. La serología (IgM) positiva para VEB no se adjudicó en ese momento como causa de la fiebre de cuatro meses de evolución. Al examen físico del ingreso el paciente se encontraba febril, con adenopatías cervicales e inguinales bilaterales y dolor abdominal difuso a la palpación profunda. Los exámenes de laboratorio al ingreso demostraron una leucocitosis de 26.600 cél $/ \mathrm{mm}^{3}$ (neutrófilos $75 \%$, linfocitos $18 \%$ ), hemoglobina $11,3 \mathrm{~g} / \mathrm{dl}$, hematocrito $35,7 \%$, plaquetas $665.000 /$ $\mathrm{mm}^{3}$ ), PCR $10 \mathrm{mg} / \mathrm{L}$, perfil hepático normal, anticuerpos antinucleares negativos y recuento de inmunoglobulinas normales para la edad.

El estudio histopatológico de los ganglios inguinales izquierdos mostró una hiperplasia linfoide con proliferación linfoidea policlonal atípica, presencia de microgranulomas y focos de micronecrosis no caseificante. El cultivo, en medio Ogawa-Kudoh a $37^{\circ} \mathrm{C}$, evidenció a las seis semanas de incubación, crecimiento de más de 100 colonias lisas, globosas y amarillentas de Mycobacterium sp. El antibiograma mostró sensibilidad a pirazinamida, kanamicina y levofloxacina, así como resistencia a la isoniacida, etambutol, rifampicina, estreptomicina y cicloserina. La identificación final por pruebas bioquímicas fue M. scrofulaceum.

Ante el informe microbiológico preliminar de $\mathrm{Myco}$ bacterium sp., se inició tratamiento antituberculoso con isoniacida $10 \mathrm{mg} / \mathrm{kg} /$ día, rifampicina $15 \mathrm{mg} / \mathrm{kg} /$ día, pirazinamida $35 \mathrm{mg} / \mathrm{kg} /$ día y etambutol $20 \mathrm{mg} / \mathrm{kg} / \mathrm{día}$, de acuerdo a la normativa ecuatoriana vigente. A los quince días de tratamiento se recibe el resultado del antibiograma, lo cual generó que se discutiera el caso en el Comité $\mathrm{Na}-$ cional de Tuberculosis, en donde se concluyó que en vista de la ausencia de contacto previo con persona infectada con tuberculosis y debido a la respuesta clínica favorable al tratamiento (tendencia a la disminución de la fiebre), se decidió continuar con el tratamiento inicial hasta tener el resultado de la tipificación. A las cinco semanas se suspendió la terapia antituberculosa debido a que el paciente presentó una reacción adversa medicamentosa con compromiso hepático, posterior a lo cual se evidenció una mejoría clínica notable con defervescencia de la fiebre y de los signos de afectación hepática, momento en el que se recibió la tipificación de la micobacteria, por lo que se dio de alta.

En los controles de seguimiento del paciente, al mes y a los tres meses de egreso, el paciente se encontraba en buen estado general, afebril y sin adenopatías palpables ni otras alteraciones al examen físico y con recuento leucocitario normal.

\section{Discusión}

Para realizar el diagnóstico del M. scrofulaceum es fundamental diferenciarlo de otras micobacterias de crecimiento lento, especialmente con Mycobacterium avium y parascrofulaceum; lo cual puede requerir el uso de pruebas moleculares o bioquímicas. En el último caso, la fuerte actividad ureasa positiva distingue al $M$. scrofulaceum de M. avium; mientras que su actividad arilsulfatasa positiva a los 14 días y telurita reductasa negativa lo distinguen del M. parascrofulaceum ${ }^{3}$.

En una revisión sistemática se reportó que $2 \%$ de las linfadenopatías cervicales causadas por micobacterias atípicas en pacientes pediátricos inmunocompetentes correspondían a $M$. scrofulaceum, siendo más frecuente cuando se asociaba con M. avium-intracellulare $(13 \%)^{4}$. Desde la década de los ochenta se ha documentado un descenso importante en la incidencia de M. scrofulaceum como agente causal de linfadenitis en niños ${ }^{5}$, probablemente asociado a la cloración del agua, su principal reservorio, y al advenimiento de métodos diagnósticos más específicos (que ha evidenciado un aumento del aislamiento del complejo M. avium en su lugar) ${ }^{6}$. En Sudáfrica, M. scrofulaceum ha llegado a ser la segunda micobacteria atípica que con mayor frecuencia se aísla en muestras pulmonares, particularmente en adultos que desarrollan actividades mineras y que presentan silicosis y pacientes con infección por $\mathrm{VIH}^{7}$.

La epidemiología de las infecciones por micobacterias atípicas en Latinoamérica difiere en los distintos estudios. La frecuencia de aislamientos de M. scrofulaceum, con respecto a las muestras analizadas, oscila entre 0 y $7,2 \%^{8-11}$. En Ecuador, los datos son limitados. Se ha descrito sólo una infección por el complejo M. aviumintracellulare-scrofulaceum en ganado bovino ${ }^{12}$, sin reportes de M. scrofulaceum en muestras humanas ${ }^{13-15}$. El caso presentado corresponde al primer reporte en humanos de esta micobacteria en nuestro país.

Entre las manifestaciones clínicas descritas para las infecciones por M. scrofulaceum, se encuentran: adenopatías cervicales unilaterales (más frecuentemente en niños 
menores de 5 años) $)^{5,16}$, parotiditis bilateral ${ }^{17}$, lesiones cutáneas (únicas o múltiples, tipo granuloma o absceso)2 ${ }^{2}$, conjuntivitis ${ }^{18}$, enfermedad pulmonar ${ }^{19}$ e intratorácica ${ }^{20}$, derrame pericárdico ${ }^{21}$, hepatitis granulomatosa ${ }^{18}$, meningitis $^{18}$, osteomielitis ${ }^{2,18}$ y enfermedad diseminada ${ }^{22}$; algunas de éstas en pacientes inmunocomprometidos con infección por $\mathrm{VIH}^{17,23}$, linfoma ${ }^{24}$, deficiencia del receptor 1 del interferón gamma ${ }^{22}$, lupus eritematoso sistémico ${ }^{2}$ y pacientes sometidos a trasplantes ${ }^{19}$.

En contraste, las infecciones por M. scrofulaceum no diseminadas se presentan con mayor frecuencia en pacientes inmunocompetentes, al igual que el caso presentado. La ausencia de antecedentes personales y familiares de alguna inmunodeficiencia primaria así como los niveles normales de inmunoglobulinas, serologías negativas para infecciones que condicionen estados de inmunocompromiso y ausencia de anticuerpos por enfermedades autoinmunes nos llevó a manejar al paciente como inmunocompetente.

Igualmente, se ha descrito el compromiso de ganglios linfáticos no cervicales por M. scrofulaceum, generalmente asociado con el complejo M. avium-intracellulare ${ }^{25}$; no obstante, esta sería la primera vez que se reporta una linfadenitis bilateral de cuello, región axilar, tórax, abdomen y región inguinal en el mismo paciente. En este sentido, las series de casos sobre adenitis por micobacterias atípicas han identificado la afectación de múltiples ganglios de una cadena ganglionar, siendo excepcionales las adenitis regionales bilaterales y generalizadas ${ }^{26,27}$. En nuestro paciente, la mayor actividad metabólica en los ganglios inguinales, próximos a la puerta de entrada, junto con el cultivo, nos confirma la infección por M. scrofulaceum; sin embargo, la generalización de las linfadenopatías podría haber correspondido a la infección coincidente del VEB. Por otro lado, el antecedente traumático previo a la presentación de linfadenitis ha sido reportado en pacientes con adenopatías no cervicales ${ }^{25}$; tal como en nuestro caso, que había tenido contacto con agua de mar.

En relación al tratamiento de las adenopatías causadas por micobacterias atípicas, se ha evidenciado que la exére- sis completa se ha asociado a mayor tasa de curación que el tratamiento médico o la no intervención ${ }^{4,16,28}$; a pesar de ello, existen situaciones en las que el tratamiento médico está indicado, como: linfadenopatías bilaterales, cuadro clínico con más de un mes de evolución, múltiples fistulizaciones, exéresis incompleta o recidiva postoperatoria, e impedimento para realizar la intervención quirúrgica (alto riesgo de complicaciones en ganglios de difícil abordaje $)^{29}$. Actualmente, el esquema de tratamiento médico empírico para micobacterias atípicas se basa en la administración de un macrólido (azitromicina o claritromicina) asociado con etambutol, ciprofloxacina, rifampicina o rifabutina, durante tres a seis meses ${ }^{4,28,29}$. Particularmente, en este caso se le realizó la exéresis de los ganglios inguinales izquierdos debido a la actividad metabólica que presentaban en la PET y a su accesibilidad, lo cual no mejoró el cuadro clínico sino hasta la administración de pirazinamida; obteniendo una respuesta favorable a un mes de tratamiento.

Agradecimientos: A la Dra. Gabriela de La Torre, por su valiosa colaboración al momento de la recolección de los datos del caso y su revisión crítica.

\section{Resumen}

Mycobacterium scrofulaceum es una micobacteria atípica de crecimiento lento que tiene como reservorio el ambiente. De forma ocasional causa enfermedad en humanos. Se presenta el caso de un niño de 10 años de edad con fiebre de cuatro meses de evolución, adenopatías cervicales, torácicas, abdominales e inguinales bilaterales, exantema, dolor abdominal y vómitos; con evidencia concomitante de serología positiva para el VEB y cultivo de los ganglios inguinales izquierdos con crecimiento de M. scrofulaceum. Es la primera comunicación de una infección causada por esta micobacteria en Ecuador, y que particularmente se presentó con un patrón de resistencia inusual.

\section{Referencias bibliográficas}

1.- Pérez-Velez C M, Kasperbauer S H, Iseman M D. Group II. Scotochromogenic Mycobacterium (M. scrofulaceum, $M$. szulgai, $M$. gordonae). Infectious Disease \& Antimicrobial Agents 2008 [citado el 21 de octubre de 2017]. Disponible en: http://www. antimicrobe.org/ms07.asp\#

2.- Valdés F, Cid A. Micobacterias atípicas. Actas Dermosifiliogr 2004; 95: 13-39 [citado el 21 de octubre de 2017]. Disponible en: http:// www.actasdermo.org/es/micobacterias-atipicas/ articulo/13064028/

3.- Bercovier H, Vincent V. Mycobacterial infections in domestic and wild animals due to Mycobacterium marinum, $M$. fortuitum, $M$. chelonae, M. porcium, M. farcinogenes, $M$. smegmatis, M. scrofulaceum, M. xenopi, $M$. kansasii, M. simiae and M. genavense. Rev Sci Tech 2001; 20: 265-90.

4.- Zimmermann P, Tebruegge M, Curtis N, Ritz N. The management of non-tuberculous cervicofacial lymphadenitis in children: a systematic review and meta-analysis. J Infect 2015; 71: 9-18. doi: 10.1016/j.jinf.2015.02.010.
5.- Wolinsky E. Mycobacterial lymphadenitis in children: a prospective study of 105 nontuberculous cases with long-term follow-up. Clin Infect Dis 1995; 20: 954-63.

6.- Griffith D E, Aksamit T, Brown-Elliott B A, Catanzaro A, Daley C, Gordin F, et al. An official ATS/IDSA statement: diagnosis, treatment, and prevention of nontuberculous mycobacterial diseases. Am J Respir Crit Care Med 2007; 175: 367-416.

7.- Hoefsloot $\mathrm{W}$, van Ingen $\mathrm{J}$, Andrejak $\mathrm{C}$, Ängeby K, Bauriaud R, Bemer P, et al. The geographic diversity of nontuberculous 
mycobacteria isolated from pulmonary samples: an NTM-NET collaborative study. Eur Respir J 2013; 42:1604-13. doi: 10.1183/09031936.00149212.

8.- Imperiale B, Zumárraga M, Gioffré A, Di Giulio B, Cataldi A, Morcillo N. Disease caused by non-tuberculous mycobacteria: diagnostic procedures and treatment evaluation in the North of Buenos Aires Province. Rev Argent Microbiol 2012; 44:3-9. doi: 10.1590/ S0325-75412012000100002.

9.- Mederos L, Fofana A, Perovani M, García G, Montoro H. Identificación de especies micobacterianas en Cuba. Arch Venez Farmacol y Ter 2007; 26:142-5.

10.- Pedro H da S, Pereira M I, Goloni M do R, Ueki SY, Chimara E. Nontuberculous mycobacteria isolated in São José do Rio Preto, Brazil between 1996 and 2005. J Bras Pneumol 2008; 34: 950-5.

11.- Ferreira R M, Saad M H, Da Silva M G, Fonseca L de S. Non-tuberculous mycobacteria I: one year clinical isolates identification in Tertiary Hospital Aids Reference Center, Rio de Janeiro, Brazil, in pre highly active antiretroviral therapy era. Mem Inst Oswaldo Cruz 2002; 97: 725-9.

12.- Proaño-Pérez F, Rigouts L, Brandt J, Dorny P, Ron J, Chávez M A, et al. Preliminary observations on Mycobacterium spp. in dairy cattle in Ecuador. Am J Trop Med Hyg 2006; 75: 318-23

13.- León M. Tipificación de micobacterias a partir de cepas inactivadas mediante analisis de patrones de restricción (PRA). Tesis de Grado. Escuala Superior Politécnica del Litoral 2015 [citado el 5 de noviembre de 2017]. Disponible en: http://www.dspace.espol.edu.ec/xmlui/ handle/123456789/29598.

14.- Paredes T. Identificación de micobacterias no tuberculosas (MNT) o atípicas por medio de la técnica de diagnóstico molecular polymerase chain reaction (PCR) and restriction enzime analisis (PRA) and restriction enzyme analysis (PRA), analisis de patrones de restricción. Tesis de Grado. Repositorio Institucional de la Universidad de las Fuerzas Armadas ESPE; 2008 [citado el 5 de noviembre de 2017]. Disponible en: http://repositorio.espe.edu.ec:8080/ handle/21000/687.

15.- Barba P, Ortega D, Espinel N, Izurieta N, Moreno F, Zurita J. Identification of nontuberculous mycobacteria isolated in Ecuador by sequencing analysis targeting $16 \mathrm{~S}$ rRNA, hsp65 and rpoB genes [Internet]. Quito; 2015 [citado el 5 de noviembre de 2017]. p. 1. Disponible en: https://www.researchgate. net/publication/295861091_Identification of_non-tuberculous_mycobacteria_isolated_ in_Ecuador_by_sequencing_analysis_ targeting_16S_rRNA_hsp65_and_rpoB_genes. DOI: $10.13140 / \mathrm{RG} .2 .2 .15195 .46889$.

16.- Zeharia A, Eidlitz-Markus T, Haimi-Cohen Y, Samra Z, Kaufman L, Amir J. Management of nontuberculous mycobacteria-induced cervical lymphadenitis with observation alone. Pediatr Infect Dis J 2008; 27: 920-2. doi: 10.1097/ INF.0b013e3181734fa3.

17.- Lawn S D, Checkley A, Wansbrough-Jones M H. Acute bilateral parotiditis caused by Mycobacterium scrofulaceum: immune reconstitution disease in a patient with AIDS. Sex Transm Infect 2005; 81: 517-8.

18.- Swanson D S, Pan X, Musser J M. Identification and subspecific differentiation of Mycobacterium scrofulaceum by automated sequencing of a region of the gene (hsp65) encoding a 65-kilodalton heat shock protein. J Clin Microbiol 1996; 34: 3151-9.

19.- Suzuki S, Morino E, Ishii M, Namkoong H, Yagi K, Asakura T, et al. Clinical characteristics of pulmonary Mycobacterium scrofulaceum disease in 2001-2011: a case series and literature review. J Infect Chemother 2016; 22: 611-6. doi: 10.1016/j.jiac.2016.06.006.

20.- Nolt D, Michaels M G, Wald E R. Intrathoracic disease from nontuberculous mycobacteria in children: two cases and a review of the literature. Pediatrics 2003; 112: e434.

21.- Singh D, Thakur K, Kalpana, Goel A. Mycobacterium scrofulaceum: an isolate from pericardial effusion. Ind J Tub 2002; 49: 7-10. [citado el 20 de noviembre de 2017]
Disponible en: http://medind.nic.in/ibr/t02/i1/ ibrt02i1p49.pdf.

22.- Marazzi M, Chapgier A, Defilippi A, Pistoia V, Mangini S, Savioli C, et al. Disseminated Mycobacterium scrofulaceum infection in a child with interferon- $\gamma$ receptor 1 deficiency. Int J Infect Dis 2010; 14: e:167-70. doi: 10.1016/j. ijid.2009.03.025.

23.- Núñez M J, Ojea R, Anibarro L, Pascual A. Infección diseminada por Mycobacterium scrofulaceum en un paciente infectado por el VIH. An Med Interna (Madrid) 2002; 19: 3812.

24.- Wang Y-W, Hung C, Tseng C-C, Chen P-J, Chao C-M. Disseminated Mycobacterium scrofulaceum infection in a patient with lymphoma. Appl Med Res 2015; 1: 81-3 [citado el 20 de noviembre de 2017]. Disponible en: http://www.scopemed.org/?mno=181115.

25.- Holland A J, Holland J, Martin H C, Cummins G, Cooke-Yarborough C, Cass D T. Noncervicofacial atypical mycobacterial lymphadenitis in childhood. J Pediatr Surg 2001; 36: 1337-40.

26.- Thoon K C, Subramania K, Chong C Y, Chang $\mathrm{K}$ T, Tee N W. Granulomatous cervicofacial lymphadenitis in children: A nine-year study in Singapore. Singapore Med J 2014; 55: 427-31.

27.- Méndez Echevarría A, Baquero Artigao F, García M M J, Romero Gómez M P, Alves Ferreira F, Del Castillo M F. Adenitis por micobacterias no tuberculosas. An Pediatr (Barc) 2007; 66: 254-9.

28.- Ruiz Del Olmo Izurquiza, Bustillo Alonso M, Monforte Cirac M L, Burgués Prades P, Guerrero Laleona C. Lindadenitis por micobacterias no tuberculosas: experiencia de 15 años. An Pediatr (Barc) 2017; 86: 115-21. doi:10.1016/j.anpedi.2016.03.006.

29.- Núñez Cuadros E, Baquero Artigao F; Grupo de trabajo sobre infección por micobacterias no tuberculosas de la SEIP. Recomendaciones de la Sociedad Española de Infectología Pediátrica sobre el diagnóstico y tratamiento de las adenitis por micobacterias no tuberculosas. An Pediatr (Barc) 2012; 77: 2018. e1-208. doi: 10.1016/j.anpedi.2012.02.018. 